\title{
Vladimir Sudakov and double-logarithmic asymptotics of amplitudes in QED, QCD and gravity
}

\author{
L. N. Lipatov ${ }^{1,2, a}$ \\ ${ }^{1}$ Petersburg Nuclear Physics Institute, Gatchina, Orlova Roshcha, 188300, St.Petersburg, Russia \\ ${ }^{2}$ Saint Petersburg State University, St. Petersburg, Russia
}

\begin{abstract}
.
We review the Sudakov results on the double logarithmic asymptotics of the electron form-factor which were based on his parametrization of the virtual particle momenta in the Feynman diagrams. The high energy amplitudes for various QED and QCD processes in the double-logarithmic approximation are obtained by using the Bethe-Salpeter approach and the evolution equations. The ultraviolet divergency of the graviton Regge trajectory allows to derive the infrared evolution equation for the graviton-graviton scattering amplitude with a double-logarithmic accuracy. The asymptotic behavior of this amplitude depends essentially on the rank $N$ of the super-symmetry.
\end{abstract}

\section{Introduction}

The investigation of scattering amplitudes at high energies $\sqrt{s}$ in QED was initiated by Vladimir Vasil'evich Sudakov 60 years ago in his paper [1], devoted to the asymptotic behavior of the vertex function in the double logarithmic approximation (DLA) valid in the region

$$
\alpha \ln ^{2} \frac{s}{m_{e}} \sim 1, \alpha=\frac{e^{2}}{4 \pi} \ll 1, s=\left(p_{e}+p_{\bar{e}}\right)^{2},
$$

where $p_{e}$ and $p_{\bar{e}}$ are momenta of initial electron and positron. Really V. Sudakov considered the case of the high energy scattering of the virtual electron off a charged center. In the case of the real electron there are infrared divergencies which can be removed by the introduction of the photon mass $\lambda$. These divergencies are canceled in the inclusive cross-section for the inelastic scattering with the emission of an arbitrary number of photons [2]. In one loop approximation the vertex function $\gamma^{(1)}$ for the $e^{+} e^{-}$ annihilation in a photon with the large virtuality $s$ can be calculated with the Sudakov parametrization of the momentum $k$ (see Figure)

$$
\begin{gathered}
k=\alpha p_{e}^{\prime}+\beta p_{\bar{e}}^{\prime}+k_{\perp}, p_{e}=p_{e}^{\prime}+\frac{m_{e}^{2}}{s^{\prime}} p_{\bar{e}}^{\prime}, p_{\bar{e}}=p_{\bar{e}}^{\prime}+\frac{m_{e}^{2}}{s^{\prime}} p_{e}, k_{\perp} p_{e}^{\prime}=k_{\perp} p_{\bar{e}}^{\prime}=0, \\
d^{4} k=\frac{\left|s^{\prime}\right|}{2} d \alpha d \beta d^{2} k_{\perp},|\alpha| \ll 1,|\beta| \ll 1, s^{\prime}=2 p_{e}^{\prime} p_{\bar{e}}^{\prime} \approx s,-k_{\perp}^{2} \ll \sqrt{s} .
\end{gathered}
$$

\footnotetext{
${ }^{\mathrm{a}}$ The investigation is supported by the grants 11.38.223.2015 of SPSU and 14-2200281 of RFBI. e-mail: lipatov@thd.pnpi.spb.ru
} 


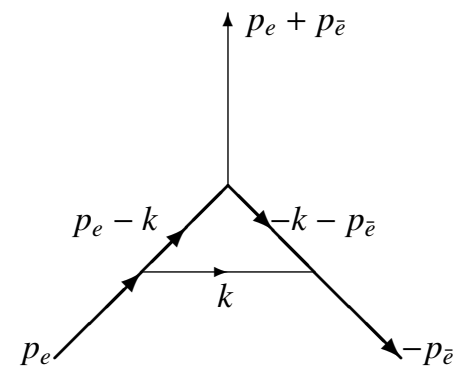

According to V. Sudakov the vertex function $\gamma^{(1)}$ after its integration over $k_{\perp}$ can be written as follows

$$
\frac{\gamma^{(1)}}{\gamma_{\text {Born }}}=-\frac{e^{2}}{8 \pi^{2}} \int_{0}^{1} \int_{0}^{1} \frac{d \alpha d \beta \theta\left(s \alpha \beta-\lambda^{2}\right)}{\left(\alpha+\frac{m_{e}^{2}}{s} \beta\right)\left(\beta+\frac{m_{e}^{2}}{s} \alpha\right)}=-\frac{e^{2} \ln \frac{s}{m_{e}^{2}}}{8 \pi^{2}}\left(\frac{\ln \frac{s}{m_{e}^{2}}}{2}+\ln \frac{m_{e}^{2}}{\lambda^{2}}\right) .
$$

One can derive the evolution equation for $\gamma$ in the infrared cut-off $\lambda$ in DLA

$$
\frac{\partial \gamma}{\partial \ln m_{e}^{2} / \lambda^{2}}=-\frac{e^{2}}{8 \pi^{2}} \ln \frac{s}{m_{e}^{2}} \gamma
$$

Its solution allows to calculate the Sudakov vertex function in the DL approximation

$$
\gamma=\gamma_{\text {Born }} \exp \left(-\frac{e^{2} \ln \frac{s}{m_{e}^{2}}}{8 \pi^{2}}\left(\frac{\ln \frac{s}{m_{e}^{2}}}{2}+\ln \frac{m_{e}^{2}}{\lambda^{2}}\right)\right) .
$$

The probability for the emission of $n$ additional soft photons obeys the Poisson distribution

$$
w_{n}=e^{-a} \frac{a^{n}}{n !}, a=2 \frac{e^{2} \ln \frac{s}{m_{e}^{2}}}{8 \pi^{2}}\left(\frac{\ln \frac{s}{m_{e}^{2}}}{2}+\ln \frac{m_{e}^{2}}{\lambda^{2}}\right),
$$

but one should take into account the energy conservation for the total frequency $\omega$ of the emitted photons. It turns out, that the QED radiative corrections to the production of narrow resonances are large. For example, the double-logarithmic result for the cross-section of the inclusive Z-boson production in the $e^{+} e^{-}$collisions at $\sqrt{s}-M_{Z} \ll M_{Z}$ has the form (cf. [3])

$$
\sigma_{e^{+} e^{-} \rightarrow Z+p h o t o n s}=\int_{0}^{M_{Z}} \sigma(W-\omega) \frac{d \omega}{\omega} \frac{4 \alpha}{\pi} \ln \frac{W}{m_{e}}\left(\frac{W}{\omega}\right)^{-\frac{4 \alpha}{\pi} \ln \frac{W}{m_{e}}}, W=\sqrt{s},
$$

where $\sigma(W)$ is the well known Born cross-section of the Z-boson production in the $e^{+} e^{-}$collisions

$$
\sigma(W)=\frac{3 \pi}{M_{Z}^{2}} \frac{\Gamma_{e \bar{e}} \Gamma_{t o t}}{\left(W-M_{Z}\right)^{2}+\frac{\Gamma_{t o t}^{2}}{4}} .
$$

Note, that $\sigma_{e^{+} e^{-} \rightarrow Z+p h o t o n s}$ does not depend on $\lambda$ and behaves as $\sim 1 /\left(W-M_{Z}\right)$ above the resonance.

\section{Backward Compton scattering and electron reggeization}

A.A. Abrikosov in ref. [2] and in subsequent papers of 1956 investigated the backward $\gamma e$ scattering at high energies and fixed momentum transfers ( $k$ and $k^{\prime}$ are initial and final momenta of the photon)

$$
s=(k+p)^{2} \gg u=\left(k^{\prime}-p\right)^{2} \sim m_{e}^{2} .
$$


He obtained the simple Sudakov-type result for the corresponding amplitude in DLA

$$
A_{D L}(s, u)=A_{B} \exp \left(-\frac{\alpha}{2 \pi} \ln \frac{s}{m_{e}^{2}} \ln \frac{|u|}{\lambda^{2}}\right) .
$$

Ten years later M. Gell-Mann with collaborators discovered, that $A(s, u)$ for fixed $u \sim \lambda^{2}$ has the form

$$
A(s, u)=A_{B}\left(\frac{s}{m_{e}^{2}}\right)^{\omega(u)}, u=-|q|_{\perp}^{2},
$$

where the Regge trajectory $\omega(\omega)$ in one loop is given below

$$
\omega(u)=\frac{\alpha}{2 \pi}\left(\hat{q}-m_{e}\right) \int \frac{d^{2} k_{\perp}}{\pi} \frac{1}{k_{\perp}^{2}-\lambda^{2}} \frac{\hat{q}_{\perp}-\hat{k}_{\perp}+m_{e}}{(q-k)_{\perp}^{2}-m_{e}^{2}} .
$$

V. G. Gorshkov, V. N. Gribov and G. V. Frolov performed similar calculations in the real case $\lambda \rightarrow 0$ and found, that the integration region $k_{\perp}^{2} \ll q^{2}$ over the virtual photon momenta gives an universal contribution and produces a divergent factor in the amplitude. The universality of this factorization for small $k_{\perp}$ was proven by V.N. Gribov with the use of the dispersion relations also for the case of the QED corrections to the strong interactions in the Regge kinematics. Below we shall apply the Gribov arguments for the derivation of the non-linear evolution equations for the amplitudes in DLA.

\section{Charge particle scattering and annihilation in QED}

The forward scattering of charged particles in QED is related to the Pomeranchuk singularity responsible for the high energy behavior of total cross-sections which does not contain double logarithms.

But one can consider the process $e^{+} e^{-} \rightarrow \mu^{+} \mu^{-}$in the kinematics when $\mu^{-}$flies along $e^{-}$

$$
s=\left(p_{e}+p_{\bar{e}}\right)^{2} \gg t=\left(p_{e}-p_{\mu}\right)^{2} \sim m^{2} .
$$

It turns out [4], that the amplitude for this process is factorized in DLA

$$
A_{D L}(s)=A_{\text {Born }} f(s), f(s)=f\left(s, p^{2}\right)_{p^{2}=m^{2}}
$$

and the main contribution to it goes from ladder diagrams satisfying the Bethe-Salpeter equation [4]

$$
f\left(s, p^{2}\right)=1+\frac{\alpha}{2 \pi} \int_{m^{2}}^{s} \frac{d s^{\prime}}{s^{\prime}} \int_{\max \left(m^{2}, p^{2} \frac{s^{\prime}}{s}\right)}^{s^{\prime}} \frac{d p^{\prime 2}}{p^{\prime 2}} f\left(s^{\prime}, p^{\prime 2}\right) .
$$

The solution of this equation allows to find $f(s)$ on the mass shell $p^{2}=m^{2}$ in DLA [4]

$$
f(s)=\int_{a-i \infty}^{a+i \infty} \frac{d j}{2 \pi i}\left(\frac{s}{m^{2}}\right)^{j} \frac{2}{j+\sqrt{j^{2}-\frac{2 \alpha}{\pi}}}=I_{0}\left(\sqrt{\frac{\alpha}{\pi}} \ln \frac{s}{m^{2}}\right) .
$$

For the $e^{+} e^{-}$backward scattering in the Regge kinematics

$$
s=\left(p_{1}+p_{2}\right)^{2} \gg u=\left(p_{1}-p_{2}^{\prime}\right)^{2} \sim m_{e}^{2}
$$

the amplitude is also factorized

$$
A_{D L}(s)=A_{\text {Born }} f(s), f(s)=f\left(s, p^{2}\right)_{p^{2}=m^{2}} .
$$


In this case apart from the ladder diagrams a large contribution appears also from the Sudakov-type photon insertions. The corresponding Bethe-Salpeter equation is more complicated but it can be solved explicitly, which allows one to obtain the corresponding amplitude on the mass shell [5]

$$
f(s)=4 \int_{a-i \infty}^{a+i \infty} \frac{d l}{2 \pi i} e^{l \rho} \frac{d}{d l} \ln D_{-\frac{1}{4}}(l), \rho=\sqrt{\frac{2 \alpha}{\pi}} \ln \frac{s}{m^{2}} .
$$

Here we omitted the Sudakov factor from soft photons with $k_{\perp} \ll m$ and used the expression

$$
D_{p}(x)=\frac{e^{-\frac{x^{2}}{4}}}{\Gamma(-p)} \int_{0}^{\infty} \frac{d t}{t^{1+p}} e^{-x t-\frac{t^{2}}{2}}
$$

for the parabolic cylinder function.

\section{Infrared evolution equations}

A simple approach for the derivation of scattering amplitudes in QED and QCD at DLA was suggested in the paper [6]. It is based on the introduction of the infrared cut-off $\mu$ at the transverse momenta of virtual particles in the Feynman diagrams

$$
\left|k_{\perp}\right|^{2}>\mu^{2}
$$

and the subsequent construction of the evolution equation in $\mu$ for the amplitudes on the mass shell. This method is similar to the renormalization group approach in the Sudakov interpretation [7].

For example, for the $e^{+} e^{-}$forward scattering the $\mu^{2}$-evolution equation can be written as follows

$$
f\left(s, \mu^{2}\right)=1+e^{2} \int_{-\infty}^{\infty} \frac{d s \alpha}{s \alpha} \frac{d s \beta}{s \beta} \int_{\mu} \frac{d^{2} k_{\perp}}{i(2 \pi)^{4}} f\left(s \alpha,\left|k_{\perp}\right|^{2}\right) \frac{\left|k_{\perp}\right|^{2}}{\left(s \alpha \beta-\left|k_{\perp}\right|^{2}\right)^{2}} f\left(s \beta,\left|k_{\perp}\right|^{2}\right) .
$$

With the transition to the $j$-representation it can be reduced to the algebraic relation

$$
f\left(s, \mu^{2}\right)=\int_{a-i \infty}^{a+i \infty} \frac{d j}{\pi i}\left(\left(-\frac{s}{\mu^{2}}\right)^{j}+\left(\frac{s}{\mu^{2}}\right)^{j}\right) \frac{f_{j}}{j}, \quad f_{j}=1+\frac{\alpha}{2 \pi} \frac{f_{j}^{2}}{j^{2}},
$$

which leads to the above result for $f(s)$ expressed in terms of the Bessel function $I_{0}(x)$.

In an analogous way the evolution equation for the backward $e^{+} e^{-}$scattering amplitude is reduced to the differential equation which can be easily solved in terms of the parabolic cylinder function [6]

$$
f_{j}=1+\frac{2 \alpha}{\pi} \frac{d}{d j} \frac{f_{j}}{j}-\frac{\alpha}{2 \pi} \frac{f_{j}^{2}}{j^{2}}, \frac{f_{j}}{j}=4 \sqrt{\frac{\pi}{2 \alpha}} \frac{d}{d l} \ln \left(e^{l^{2} / 4} D_{-1 / 4}(l)\right) .
$$

In this equation the non-linear term corresponds to the ladder contribution and the term with the derivative describes the soft Sudakov photons attached to the external legs according to the Gribov factorization theorem discussed above.

In QCD the large logarithms of $Q^{2}$ in the electron-proton scattering are summed with the help of the DGLAP equation [8]. At $x \rightarrow 0$ this equation for a non-singlet parton distribution

$$
\frac{d}{d \ln Q^{2}} n_{q}\left(Q^{2}, x\right)=\frac{n_{c}^{2}-1}{2 N_{c}^{2}} \frac{\alpha_{c}\left(Q^{2}\right)}{2 \pi} \int_{x}^{1} \frac{d \beta}{\beta} n_{q}\left(Q^{2}, \beta\right)
$$


can be modified by a simple deformation of the integration region

$$
n_{q}\left(Q^{2}, x\right)=\delta(x-1)+\frac{n_{c}^{2}-1}{2 N_{c}^{2}} \frac{\alpha_{c}}{2 \pi} \int_{x}^{1} \frac{d \beta}{\beta} \int_{m^{2}}^{Q^{2} \frac{\beta}{x}} \frac{d k^{2}}{k^{2}} n_{q}\left(k^{2}, \beta\right) .
$$

Its solution corresponding to the non-singlet quark distribution in DLA is given below

$$
n_{q}\left(Q^{2}, x\right)=\int_{a-i \infty}^{a+i \infty} \frac{d j}{2 \pi}\left(\frac{1}{x}\right)^{j} \frac{j \gamma(j)}{\frac{N_{c}^{2}-1}{2 N_{c}} \frac{\alpha}{2 \pi}}\left(\frac{Q^{2}}{m^{2}}\right)^{\gamma(j)}, j \gamma(j)=\frac{N_{c}^{2}-1}{2 N_{c}} \frac{\alpha}{2 \pi}+\gamma^{2}(j) .
$$

In a similar way one can modify and solve the equations at $x \rightarrow 0$ for the singlet quark and gluon distributions in DLA [9].

\section{Graviton Regge trajectory and scattering amplitudes in super-gravity}

The graviton Regge trajectory in LLA was calculated many years ago [10]

$$
\omega\left(q^{2}\right)=\frac{\alpha}{\pi} \int \frac{q^{2} d^{2} k}{k^{2}(q-k)^{2}} f(k, q), \alpha=\frac{\kappa^{2}}{8 \pi^{2}},
$$

where $\gamma=\frac{\kappa^{2}}{8 \pi}$ is the Newton constant and

$$
f(k, q)=(k, q-k)^{2}\left(\frac{1}{k^{2}}+\frac{1}{(q-k)^{2}}\right)-q^{2}+\frac{N}{2}(k, q-k) .
$$

Here $N$ is the number of different gravitinos appearing in the corresponding action

$$
S_{3 / 2}=-\frac{1}{2} \epsilon^{\mu \nu \rho \sigma} \int d^{4} x \sum_{r=1}^{N} \bar{\psi}_{\mu}^{r} \gamma_{5} \gamma_{\nu} \partial_{\rho} \psi_{\sigma}^{r} .
$$

The graviton Regge trajectory contains infrared and ultraviolet divergencies

$$
\omega\left(q^{2}\right)=-\alpha|q|^{2}\left(\ln \frac{|q|^{2}}{\lambda^{2}}+\frac{N-4}{2} \ln \frac{|\Lambda|^{2}}{|q|^{2}}\right) .
$$

The ultraviolet divergency is fictive, because in one loop the gravity is a renormalizable theory. The cut-off $\Lambda^{2}$ should be substituted by $s$, which leads to an appearance of double-logarithmic terms in the corresponding scattering amplitude. The summation of all such terms is performed below [10].

It is convenient to use the Mellin representation of the graviton-graviton scattering amplitude

$$
A(s, t)=A_{\text {Born }} s^{-\alpha|q|^{2} \ln \frac{|q|^{2}}{\lambda^{2}}} \Phi(\xi), \Phi(\xi)=\int_{a-i \infty}^{a+i \infty} \frac{d \omega}{2 \pi i \omega}\left(\frac{s}{|q|^{2}}\right)^{\omega} f_{\omega} .
$$

The partial wave $f_{\omega}$ satisfies the infrared evolution equation similar to the QCD case [11]

$$
f_{\omega}=1+b \frac{d}{d \omega} \frac{f_{\omega}}{\omega}-b \frac{N-6}{2} \frac{f_{\omega}^{2}}{\omega^{2}}, b=\alpha|q|^{2}, \alpha=\frac{\kappa^{2}}{8 \pi^{2}}, \xi=\alpha|q|^{2} \ln ^{2} \frac{s}{|q|^{2}} .
$$

Its solution in the perturbation theory is

$$
\Phi(\xi)=1-\frac{N-4}{2} \frac{\xi}{2}+\frac{(N-4)(N-3)}{2} \frac{\xi^{2}}{4 !}-\frac{N-4}{8}\left(5 N^{2}-26 N+36\right) \frac{\xi^{3}}{6 !}+\ldots
$$


The analytic solution of the equation can be constructed in terms of the parabolic cylinder function

$$
\frac{f_{\omega}^{(N)}}{\omega}=\frac{2}{6-N} \frac{1}{\sqrt{b}} \frac{d}{d x} \ln d^{(N)}(x), d^{(N)}(x)=e^{\frac{x^{2}}{4}} D_{\frac{6-N}{2}}(x), x=\frac{\omega}{\sqrt{b}} .
$$

One can verify [11], that the quantity $A(s, t) / A_{B o r n}$ grows with energy for $N<4$ and tends to zero at $s \rightarrow \infty$ for $N>4$.

\section{References}

[1] V. V. Sudakov, Sov. Phys. JETP 3087 (1956).

[2] A. A. Abrikosov, Sov. Phys. JETP 3096 (1956).

[3] Ya. I. Azimov, V. A. Khoze, L. N. Lipatov, JETP Letters 21378 (1975).

[4] V. G. Gorshkov, V. N. Gribov, G. V. Frolov, L. N. Lipatov, Phys. Lett. 22671 (1966).

[5] V. G. Gorshkov, V. N. Gribov, L. N. Lipatov, G. V. Frolov, Yad. Fiz. 6361 (1967).

[6] R. Kirschner, L. N. Lipatov, Nucl. Phys. 123122 (1983).

[7] V. V. Sudakov, Sov. Phys. JETP 31729 (1956).

[8] V. N. Gribov, L. N. Lipatov, Sov. J. Nucl. Phys. 18 (1972) 438, 675;

L. N. Lipatov, Sov. J. Nucl. Phys. 20 (1975) 93;

G. Altarelli and G. Parisi, Nucl. Phys. B126 (1977) 298;

Yu. L. Dokshitzer, Sov. Phys. JETP 46 (1977) 641.

[9] B. L. Ioffe, V. S. Fadin, L. N. Lipatov, Quantum Chromodynamics, Perturbative and nonperturbative aspects, Cambridge University Press, (2010).

[10] L. N. Lipatov, Phys. Lett. 116, 411 (1982).

[11] J. Bartels, L. N. Lipatov, A. Sabio Vera, J. High Energy Phys. 07056 (2014). 